\title{
Prediction of the Aging of HTV Silicone Rubber Using Chemical Concentration and Polynomial Interpolation Approach
}

\author{
Ghazwan Haddad ${ }^{1}$, Khoi Loon Wong ${ }^{1}$, Rahul Gupta ${ }^{2}$ and Muthu Pannirselvam ${ }^{2}$ \\ 1. School of Electrical and Computer Engineering, RMIT University, Melbourne Melbourne VIC 3001, Australia \\ 2. School of Civil, Environmental and Chemical Engineering, RMIT University, Melbourne VIC 3001, Australia
}

Received: September 19, 2014 / Accepted: March 23, 2015 / Published: May 31, 2015.

\begin{abstract}
Predicting the lifetime of polymeric insulators is one of the most important research topics in studying the life cycle of high voltage insulators in the power transmission and distribution networks. HTV (high temperature vulcanized) silicone rubber is a high performance dielectric material used within electrical power equipment, in particular transmission and distribution insulators. In this paper, we proposed a new approach using the Newton's method and Lagrange method to predict the aging of HTV silicone rubber that are subjected to multiple stress conditions. Concentration of chemical elements such as carbon, oxygen, silicon and aluminum were obtained and evaluated using a SEM (scanning electron microscope) with EDS (energy dispersive X-ray spectroscopy). Curve fitting using the Newton's and Lagrange interpolation methods yield useful linear interpolation equations that describe the aging characteristic of the specimens under study. This approach can be applied to predict the change in chemical concentration of polymeric insulators over the life cycle with good accuracy.
\end{abstract}

Key words: Polymeric insulators, accelerated weathering tester, scanning electron microscope, Newton interpolation, Lagrange interpolation.

\section{Introduction}

The use of polymer insulator are expanding in high voltage insulation application, mainly due to their lightweight, easy installation and resistance to extreme weather conditions compared to traditional inorganic materials [1]. HTV (high temperature vulcanized) silicone rubber has been used as polymeric housing for high voltage insulators in power transmission and distribution systems for over 30 years. HTV silicone rubber has high dielectric strength and excellent thermo-mechanical properties [2] and it is regarded as one of the most used materials due to its excellent electrical, mechanical and chemical performance with

Corresponding author: Khoi Loon Wong, Associate Professor, research fields: power transmission and distribution systems, dielectric and insulation breakdown, condition monitoring of aging infrastructure, pole-top fire mitigation technique. E-mail: alan.wong@rmit.edu.au. superior aging characteristic $[2,3]$.

In Refs. [4-8], most of the works focused on the chemical characterization of specimens that gathered from the field using ATR-FTIR (attenuated total reflection-Fourier transform infrared spectroscopy), XPS (X-ray photoelectron spectroscopy) and SEM (scanning electron microscope). However, the aging of specimens in the field are subjected to many uncontrollable varying weathering factors and not one sample is identical. Moreover, different degree of aging is often observed on the same insulator depending on the location and orientation of the samples being taken.

This paper studies the aging characteristics of HTV silicone rubber under controlled environmental conditions using Accelerated Weathering Tester (Q-LAB Corporation, Westlake, USA) based on ASTM (American Society for Testing and 
Materials)-G154. Specimens are subjected to stresses such as heat, ultraviolet radiation and condensation. The percentage quantity for chemical elements in the surface was computed using Philips XL-30 SEM with EDS (energy dispersive X-ray spectroscopy). The results are used to formulate the interpolation equations using both Newton's and Lagrange interpolation methods. These equations can be applied as a prediction models for estimating the change in chemical concentration and the aging of polymeric insulators over the whole life cycle.

\section{Experimental Procedure}

This section covers the details about specimen's preparation, test procedures using SEM.

\subsection{Aged Specimens under Condensation Cycle}

To evaluate the effectiveness of the condensation method, three specimens were removed from the insulators sheds. These specimens were aged inside QUV weathering tester using cycle D (ASTM G154 cycle 4) in QUV/spray model. This cycle contains two steps. Firstly, the specimens were aged under a constant temperature of $70{ }^{\circ} \mathrm{C}$ and an ultraviolet source type A (UVA-340 nm) with a radiation level of $1.55 \mathrm{~W} / \mathrm{m}^{2}$ for $8 \mathrm{~h}$ with different exposure times $(1,000$ $\mathrm{h}, 2,000 \mathrm{~h}$ and 3,000 h). Secondly, the specimens were aged under condensation for $4 \mathrm{~h}$ with a constant temperature $50{ }^{\circ} \mathrm{C}$ without any ultraviolet source.

\subsection{SEM}

The percentage quantity for chemical elements on the surface of the specimens was computed using Philips XL-30 SEM with EDS that have silicon drift detector with X-ray detector model 500 Apollo X (EDAX (Energy Dispersive Analysis X-ray) Inc., Berwyn, USA). This type of detector provides a low relative statistical error for the element peak intensity. The specimens were coated with carbon before examination, using a Gatan Inc. precision etching and coating system (Model 682) with thickness monitor
(Model 681.2). Before examination, all specimens were cleaned in an ultrasonic cleaner for $180 \mathrm{~s}$ using ethanol.

\section{Newton and Lagrange Methods}

\subsection{Interpolation Model based on Newton Method}

Newton's interpolation polynomial is among the most popular and useful curve fitting methods for expressing an interpolating polynomial. Before presenting the general equation that used in this paper, a first order (linear interpolation) will be introduced. Linear interpolation is a simplest form of interpolation that connects two data points with straight line as depicted graphically in Fig. 1 [9].

The linear interpolation was representing the slope of the line connecting the two points as shown in Eq. (2). The term $\frac{f\left(x_{2}\right)-f\left(x_{1}\right)}{x_{2}-x_{1}}$ is a finite difference approximation of the first derivative. In general, the smaller interval between the data points, the better the approximation. Eq. (2) can be rewritten as shown in Eq. (3). The Newton's formula can be generalized to fit an $(n-1)$ th-order polynomial to $\mathrm{n}$ data points, as shown in Eq. (6):

$$
\begin{gathered}
\frac{f_{1}(x)-f\left(x_{1}\right)}{x-x_{1}}=\frac{f\left(x_{2}\right)-f\left(x_{1}\right)}{x_{2}-x_{1}} \\
f_{1}(x)=f\left(x_{1}\right)+\frac{f\left(x_{2}\right)-f\left(x_{1}\right)}{x_{2}-x_{1}}\left(x-x_{1}\right) \\
f_{1}(x)=b_{1}+b_{2}\left(x-x_{1}\right)
\end{gathered}
$$

where:

$$
\begin{gathered}
b_{1}=f(x) \\
b_{2}=\frac{f\left(x_{2}\right)-f\left(x_{1}\right)}{x_{2}-x_{1}} \\
f_{n-1}(x)=b_{1}+b_{2}\left(x-x_{1}\right)+\cdots+b_{n}\left(x-x_{1}\right)\left(x-x_{2}\right) \ldots\left(x-x_{n-1}\right)
\end{gathered}
$$

\subsection{Interpolation Model based on Lagrange Method}

Lagrange method is another method that can be used to model the aging of silicone rubber based on the chemical concentration of silicone rubber. Lagrange 


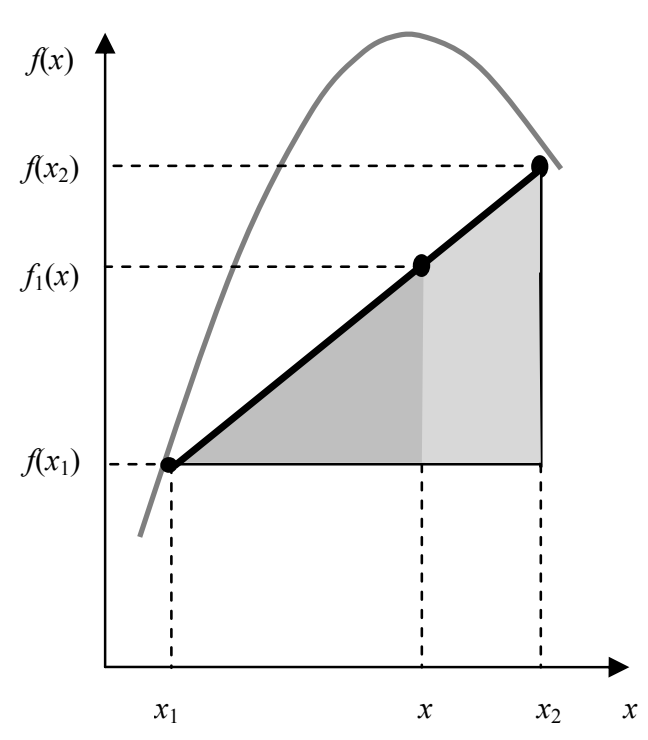

Fig. 1 Graphical depiction of linear Newton interpolation.

interpolating polynomial equation can be presented as the average of the two values which are connected by a straight line.

$$
f(x)=L_{1} f\left(x_{1}\right)+L_{2} f\left(x_{2}\right)
$$

where, the $L_{1}$ and $L_{2}$ are the weighting coefficients [9].

A linear interpolation is depicted graphically in Fig. 2. The weighting coefficients $\left(L_{1}\right.$ and $\left.L_{2}\right)$ yield the straight line that connects the points [9].

Where:

$$
\begin{aligned}
& L_{1}=\frac{x-x_{2}}{x_{1}-x_{2}} \\
& L_{2}=\frac{x-x_{1}}{x_{2}-x_{1}}
\end{aligned}
$$

The equation, as in Eq. (10) explained the predication value in any point along $f(x)$ as shown in Fig. 2.

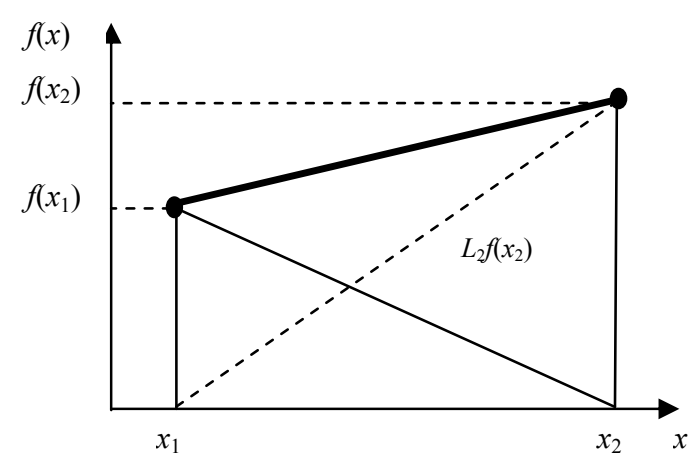

Fig. 2 Graphical depiction of linear Lagrange interpolation.
Lagrange general formula can be written as shown in Eq. (11) where $L_{i}(x)$ was represent a high order Lagrange coefficient [9].

$$
\begin{gathered}
f(x)=\frac{x-x_{2}}{x_{1}-x_{2}} f\left(x_{1}\right)+\frac{x-x_{1}}{x_{2}-x_{1}} f\left(x_{2}\right) \\
f_{n-1}(x)=\sum_{i=1}^{n} L_{i}(x) f\left(x_{i}\right)
\end{gathered}
$$

\section{Results and Discussion}

\subsection{Elements Analysis Using EDS}

Elements analysis was performed on the new and aged specimens. The percentage of the chemical elements on the silicone rubber surface was computed using Philips XL-30 SEM with EDS from EDAX Inc. Figs. 3- 6 show the atomic composition in percentage.

The data points show the average of chemical percentage taken from six locations on the silicone rubber surface under four different aging conditions

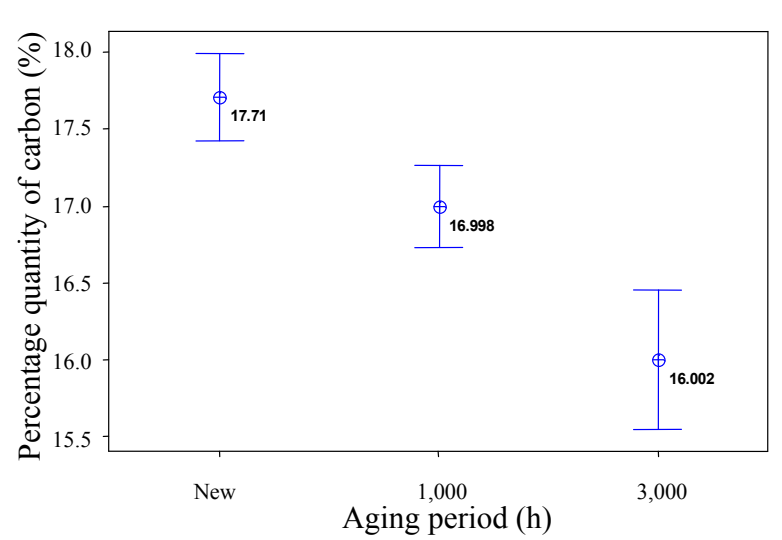

Fig. 3 Interval plot of carbon element.

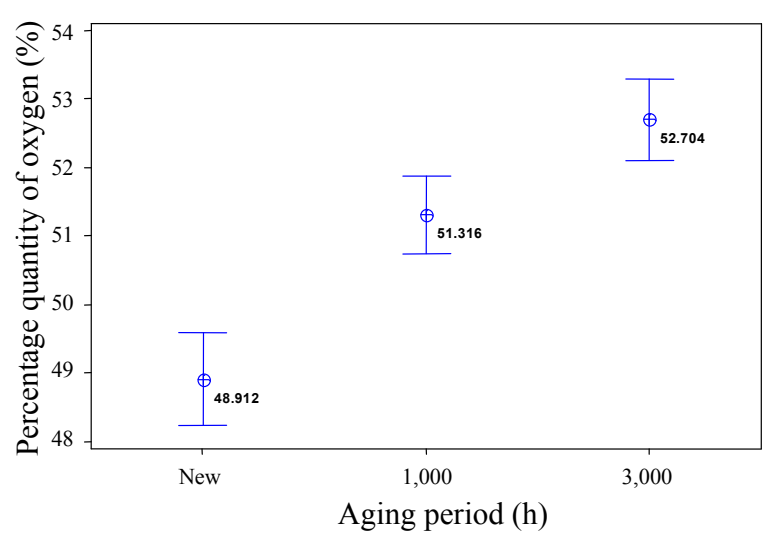

Fig. 4 Interval plot of oxygen element. 


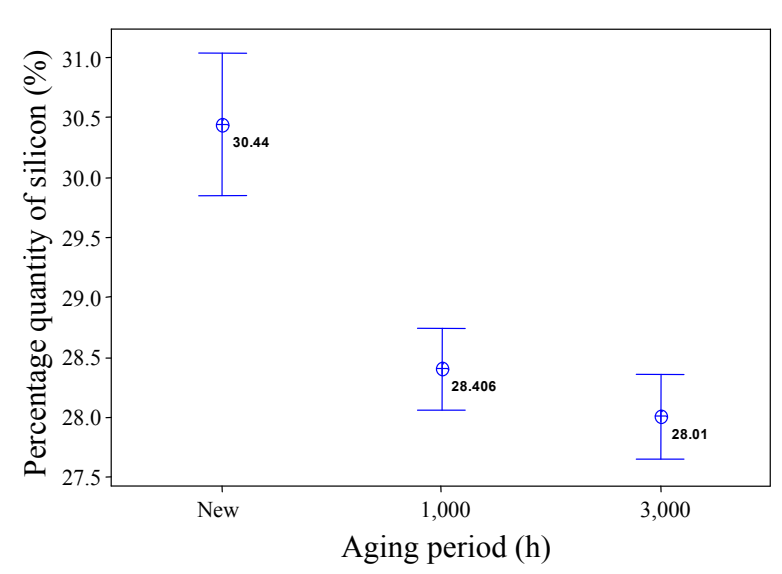

Fig. 5 Interval plot of silicon element.

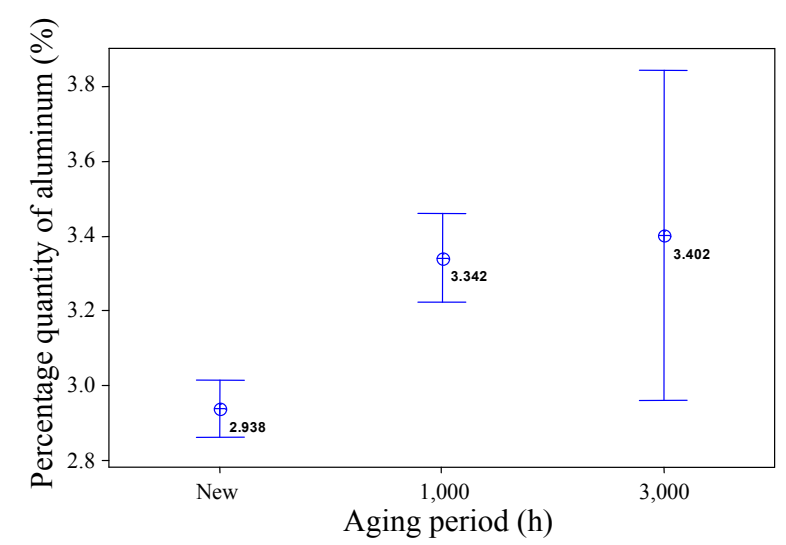

Fig. 6 Interval plot of aluminum element.

and the results are plotted in interval plot showing the maximum, minimum and average value. As clearly shown in Figs. 3-6, only limited aging data is available (namely $0,1,000 \mathrm{~h}$ and $3,000 \mathrm{~h}$ ). The interpolation methods that will be applied in the next section will be used to predict the chemical composition for other aging period.

Carbon, oxygen, silicon and aluminum elements are the main elements found in the silicone rubber where carbon and oxygen are organic elements. The most significant depletion in both aging period (new and $3,000 \mathrm{~h}$ ) was evident in the carbon, where the content changes from $17.710 \%$ to $16.002 \%$ as shown in Fig. 3 . Oxygen element increases from $48.912 \%$ to $52.704 \%$ in Fig. 4. For all aged samples, reduction of carbon percentage and increases of oxygen percentage indicates clearly that the surface oxidation of the silicone rubber took place.
4.2 Interpolation Curves from Newton and Lagrange Methods

Figs. 7-10 show the interpolation curves based on Newton and Lagrange methods. Based on these curve fitting methods, the chemical composition for the rest of the aging period can be estimated. For instance, the percentage quantity of carbon at $1,500 \mathrm{~h}$ and $2,000 \mathrm{~h}$ can be estimated to be $16.696 \%$ and $16.429 \%$ from Fig. 7. The same can be applied to other elements such as oxygen, silicon and aluminum.

The coefficients of the Newton's and Lagrange equations are tabulated in Table 1. In addition, both Newton's and Lagrange interpolation methods generated the same equations and the interpolation curves are identical.

In order to examine the effectiveness of these

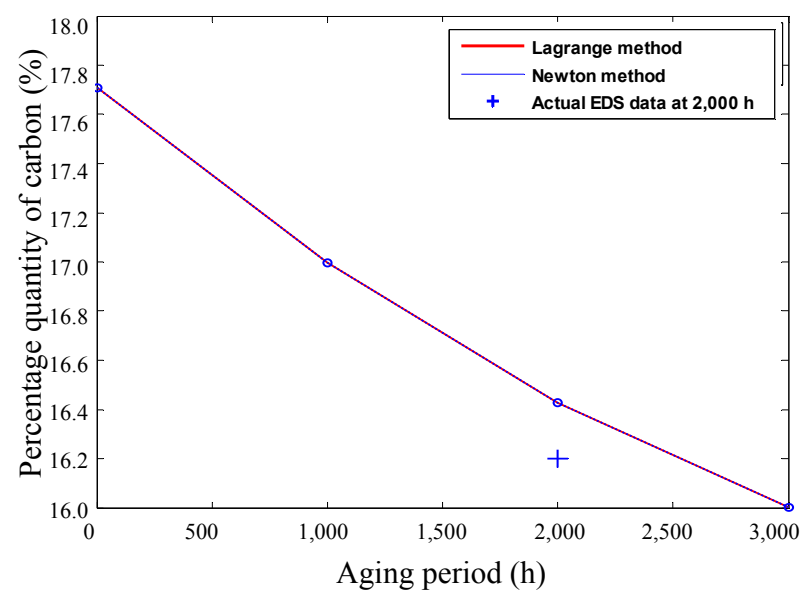

Fig. 7 Interpolation curve of carbon element.

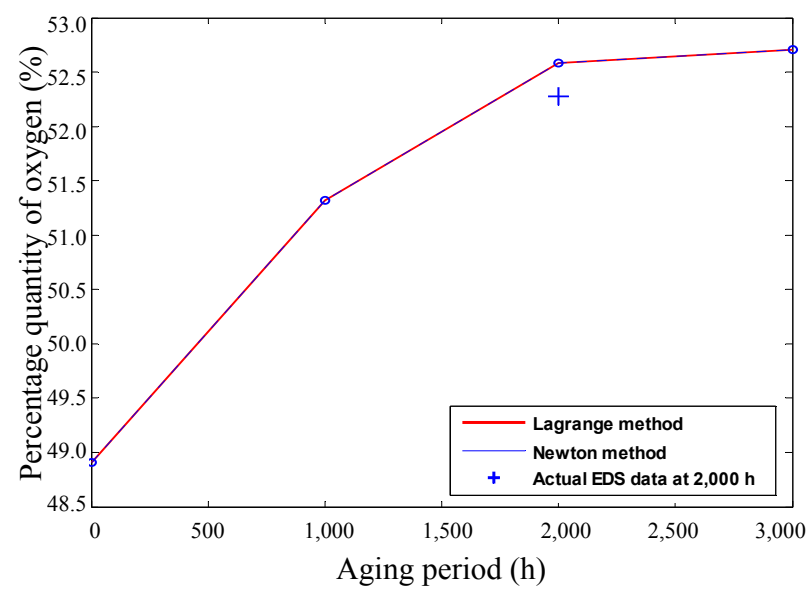

Fig. 8 Interpolation curve of oxygen element. 

and Polynomial Interpolation Approach

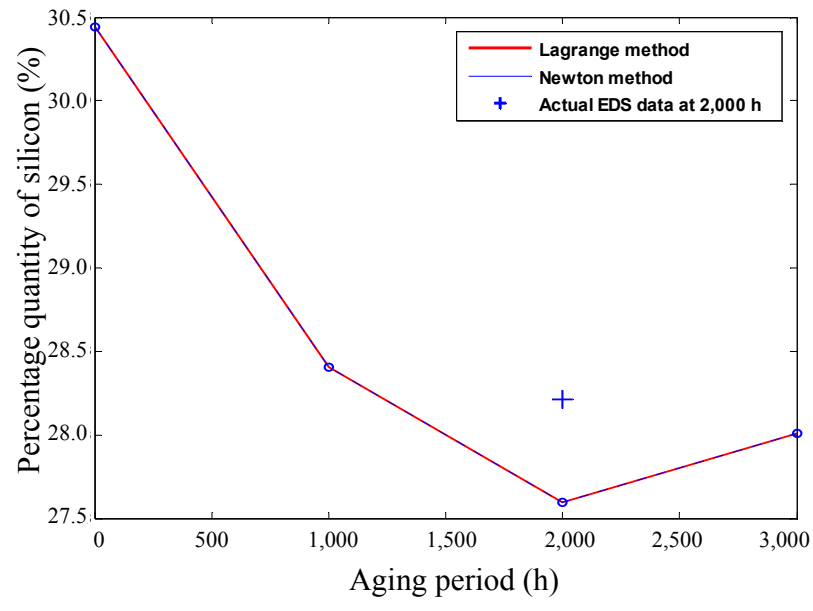

Fig. 9 Interpolation curve of silicon element.

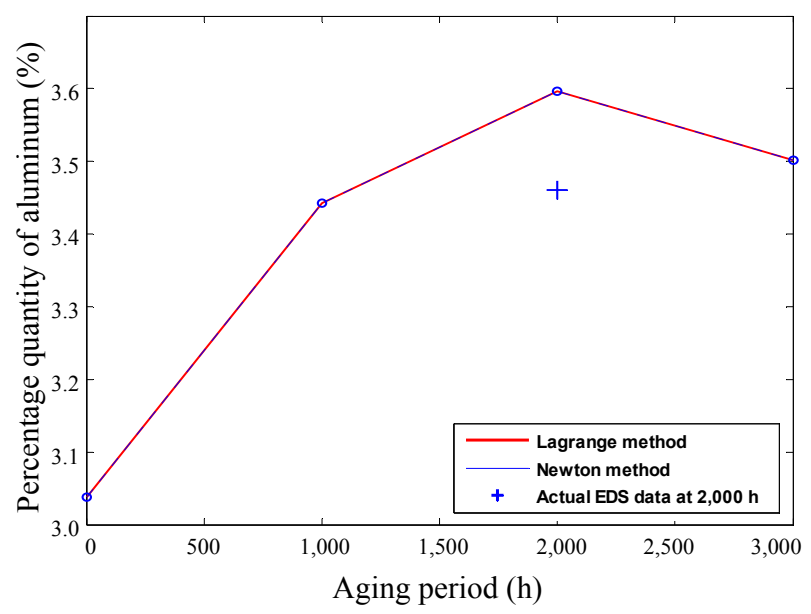

Fig. 10 Interpolation curve of aluminum element. interpolation equations, the differences between the estimated value and the actual measured value for the specimen aged for 2,000 h using cycle D (ASTM G154 cycle 4) are presented in Table 2. The actual measurement of the 2,000 h specimen was also plotted in Figs. 7-10. The percentage error between the approximated value (from Newton and Lagrange equations) and actual measurement are also presented. The small percentage error shows in Table 2 suggests that the proposal approach can be used as a tool to estimate the aging of silicone rubber. Both the Newton and Lagrange methods produced the same percentage error in estimating the data at $2,000 \mathrm{~h}$ due to the small sample size in this study.

\section{Conclusions}

The results in this paper show that, there is clear trend developed in the interval plots of the percentage chemical composition of the aged specimens based on the measurements obtained from EDS. Polynomial interpolation equations that approximate the aging profile of silicone rubber are obtained using Newton's and Lagrange methods. In order to evaluate the effectiveness of interpolation equations, chemical

Table 1 Coefficients of the Newton's and Lagrange equations for $2,000 \mathrm{~h}$ specimen.

\begin{tabular}{lllll}
\hline Coefficients of Newton polynomial equation & Carbon & Oxygen & Silicon & Aluminium \\
\hline B1 & 17.710 & 48.912 & 30.440 & 2.938 \\
B2 & $-7.12 \mathrm{e}-04$ & 0.002404 & -0.002034 & $4.04 \mathrm{e}-04$ \\
B3 & $7.13 \mathrm{e}-08$ & $-5.70 \mathrm{e}-07$ & $6.12 \mathrm{e}-07$ & $-1.25 \mathrm{e}-07$ \\
\hline Coefficients of Lagrange polynomial equation & Carbon & Oxygen & Silicon & Aluminium \\
\hline L1 & -0.333 & -0.333 & -0.333 & -0.333 \\
L2 & 1 & 1 & 1 & 1 \\
L3 & 0.333 & 0.333 & 0.333 & 0.333 \\
\hline
\end{tabular}

Table 2 Chemical Percentage for 2000 hour Specimen.

\begin{tabular}{lllll}
\hline $\begin{array}{l}\text { Chemical percentage } \\
\text { elements }\end{array}$ & $\begin{array}{l}\text { Average results from } \\
\text { EDS }\end{array}$ & $\begin{array}{l}\text { Calculation using } \\
\text { Lagrange method }\end{array}$ & $\begin{array}{l}\text { Calculation using Newton } \\
\text { method }\end{array}$ & Percentage error (\%) \\
\hline Carbon & 16.200 & 16.429 & 16.429 & 1.41 \\
Oxygen & 52.274 & 52.580 & 52.580 & 0.58 \\
Silicon & 28.212 & 27.596 & 27.596 & 2.18 \\
Aluminum & 3.360 & 3.496 & 3.496 & 4.04 \\
\hline
\end{tabular}


composition of a specimen aged for 2,000 $\mathrm{h}$ using cycle D (ASTM G154 cycle 4) are compared to the approximated values obtained using the interpolation equations. The results in this paper suggest that, the proposed approach could be applied to predict the change in chemical concentration of polymeric insulators over the life cycle. However, more investigations involving extended aging period and other insulating materials should be carried out in the future.

\section{Acknowledgments}

The authors acknowledge the facilities, and the scientific and technical assistance, of the Australian Microscopy \& Microanalysis Research Facility at the RMIT Microscopy \& Microanalysis Facility, RMIT University.

\section{References}

[1] Kikuchi, T., Nishimura, S., Nagao, M., Izumi, K., Kubota, Y., and Sakata, M. 1999. "Survey on the Use of Non-ceramic Composite Insulators." IEEE Transactions on Dielectrics and Electrical Insulation 6 (5): 548-56.

[2] Bernstorf, R. A., Niedermier, R. K., and Winkler, D. S. 2000. Polymer Compounds Used in High Voltage Insulators. Centralia: Hubbell Power Systems.

[3] Reynders, J. P., Jandrell, I. R., and Reynders, S. M. 1999.
"Review of Aging and Recovery of Silicone Rubber Insulation for Outdoor Use." IEEE Transactions on Dielectrics and Electrical Insulation 6 (5): 620-31.

[4] Umeda, I., Tanaka, K., Kondo, T., Kondo, K., and Suzuki, Y. 2008. "Acid Aging of Silicone Rubber Housing for Polymer Insulators." In Proceedings of the ISEIM (International Symposium on Electrical Insulating Materials), 518-21.

[5] Vasudev, N., Vasudevan Nambudri, P. V., Dinesh, M. N., Ravi, K. N., and Krishnan, V. 2009. "Long Term Ageing Performance of Silicone Rubber Insulators under Different Conditions." In Proceedings of the IEEE (Institute of Electrical and Electronics Engineers) 9th ICPADM (International Conference on the Properties and Applications of Dielectric Materials), 276-80.

[6] El-Hag, A. H., Jahromi, A. N., and Jayaram, S. 2007. "Aging Performance of ATH based RTV Insulator Coatings." In Proceedings of the IEEE ICSD (International Conference on Solid Dielectrics), 172-5.

[7] Hongwei, C., Dong, Y., Jinhua, H., Huan, R., Ming, L., Zhongbin, L., and Huihao, G. 2012. "Investigation and Corroboration of a Novel Method to Estimate the Hydrophobicity of Composite Insulators." IEEE Transactions on Dielectrics and Electrical Insulation 19 (6): 2029-36.

[8] Bin, M., Gubanski, S. M., and Hillborg, H. 2011. "AC and DC Zone-Induced Ageing of HTV Silicone Rubber." IEEE Transactions on Dielectrics and Electrical Insulation 18 (6): 1984-94.

[9] Steven, C. C. 2008. Applied Numerical Methods with MATLAB for Engineers and Scientists. Boston: McGraw-Hill Higher Education. 